Bulletin d'Histoire Contemporaine de I'Espagne

$50 \mid 2016$

Les intellectuels en Espagne, de la dictature à la démocratie (1939-1986)

\title{
Los intelectuales en la Transición
}

Les intellectuels au cours de la Transition

The intellectuals during the Transition

\section{Antonio García Santesmases}

\section{CpenEdition}

\section{Journals}

Edición electrónica

URL: http://journals.openedition.org/bhce/521

DOI: 10.4000/bhce.521

ISSN: 1968-3723

Editor

Presses Universitaires de Provence

Edición impresa

Fecha de publicación: 1 diciembre 2016

Paginación: 63-73

ISSN: 0987-4135

Referencia electrónica

Antonio García Santesmases, «Los intelectuales en la Transición », Bulletin d'Histoire Contemporaine de I'Espagne [En línea], 50 | 2016, Publicado el 09 octubre 2018, consultado el 22 septiembre 2020. URL: http://journals.openedition.org/bhce/521 ; DOI : https://doi.org/10.4000/bhce.521 


\section{Los intelectuales en la transición}

\section{Antonio GARCíA SANTESMASES}

UNED

$\mathrm{U}$ na primera advertencia viene por el período que vamos a estudiar, en el que se suscitan una serie de debates intelectuales de gran calado donde algunos participamos. Por ello, es difícil hacer compatibles dos aspectos que están inexorablemente presentes en nuestro tema: tener en cuenta lo que entonces dijimos, las posiciones que sustentábamos y, a la vez, intentar mirarnos desde fuera y ser capaces de ver las diferencias entre aquellos tiempos y éstos que estamos viviendo.

En el período que vamos a estudiar creo que podemos diferenciar, al menos, dos etapas. La primera abarcaría desde el 20 de noviembre de 1975 (muerte de Franco) hasta el 28 de octubre de 1982 (victoria del PSOE en las elecciones generales). Un segundo período abarcaría desde el triunfo electoral del PSOE hasta marzo de 1986 (referéndum sobre la permanencia de España en la OTAN).

Algunos consideran que la transición se debe ceñir a los años que abarcan desde la muerte del dictador hasta la aprobación de la Constitución de 1978; otros prefieren alargar el proceso hasta la victoria del PSOE en las elecciones y somos pocos los que consideramos que es preferible considerar que la transición se cierra con la entrada en la Comunidad económica europea y con la ratificación de nuestra permanencia en la OTAN ${ }^{1}$.

¿Cómo intervienen los intelectuales en todo este proceso? Voy a operar en mi texto estableciendo una diferenciación entre varios tipos de respuestas intelectuales: 1) la respuesta conservadora; 2) la interpretación liberal; 3) los avatares de la perspectiva socialista y 4) la emergencia de una izquierda alternativa. En cada uno de estos universos habitan intelectuales relevantes, medios de comunicación, editoriales, centros de investigación, todos los cuales condicionan las respuestas de los actores políticos y son a su vez condicionados por los requerimientos de los Partidos políticos, de las Iglesias, de los Sindicatos, y de los movimientos sociales.

\section{La respuesta conservadora}

Para tener en cuenta lo ocurrido en el mundo liberal-conservador hay que partir de un hecho: durante años (y especialmente entre 1982 y 1990) asistimos a una enorme crisis en el mundo de la representación política de la derecha española. Cuando hoy se recuerdan los años de la transición y se tiende a enaltecer la imagen de algunos líderes del período

1 Sobre el tema del final de la transición desarrollo mi pensamiento en A.G. SANTESMASES, Repensar la izquierda, Barcelona, Anthropos, 1993. Ver también Rafael Del Aguila (ed), Los intelectuales y la politica, Madrid, Editorial Pablo Iglesias, 2003. En el volumen se incluye mi trabajo «Cinco funciones para los intelectuales de izquierda» (p. 85-124). 
como Adolfo Suárez ${ }^{2}$, se olvidan los errores cometidos que tuvieron un enorme efecto en el mundo intelectual. Las primeras elecciones abonaban la posibilidad de un partido de centroderecha con un gran respaldo electoral, cercano a la mayoría absoluta, que tenía como tarea conducir el proceso de transición y lograr la síntesis ideológica entre los reformistas del franquismo y aquellos sectores de la oposición moderada a la dictadura que habían decidido sumarse a la Unión de Centro Democrático (UCD). Si Adolfo Suárez logró llevar a buen puerto la primera de las tareas -legalizar al partido comunista, negociar los contenidos de la Constitución, articular la salida a la crisis económica, mediante los Pactos de la Moncloa- fue incapaz de lograr esa síntesis que hubiera permitido larga vida a la UCD.

Como sabemos todo acabó en 1982 en un estallido multipolar que provocó que el propio Suárez se presentara a las elecciones por el CDS, los sectores democristianos emigraran a Alianza Popular (AP), mientras los llamados socialdemócratas de Fernández Ordóñez se coaligaban con el PSOE. Esto provoca que en el mundo posterior a 1982 cuaje una relación verdaderamente asimétrica entre el gobierno del PSOE y la oposición de Alianza Popular. El mundo conservador tardará años -tendrá que esperar a la llegada de una nueva generación- en recuperarse. A partir de la caída del muro de Berlín los vientos liberalconservadores españoles podrán enlazar con lo ocurrido en Europa y en Estados Unidos a partir de la impronta de Karol Wojtila, de Margaret Thatcher y de Ronald Reagan; pero para recoger esos frutos hay que esperar a los años noventa y analizar un periodo que va más allá del tema que nos ocupa en estas jornadas.

En el período que analizamos, la crisis en la representación política del centroderecha provoca que el mundo conservador se haga hegemónico en detrimento de las posiciones de centro progresista; esa hegemonía se traduce en la necesidad de cargar con el franquismo sociológico y de aparecer públicamente liderado por figuras representativas del franquismo político. Las repercusiones en el mundo intelectual son de un gran calado. Los representantes más genuinos del franquismo ideológico rompen con AP y deciden no apoyar la Constitución del 78. El ejemplo más relevante de esta respuesta intelectual sería la figura de Gonzalo Fernández de la Mora. En su libro de memorias Rio Arriba ${ }^{3}$ da cuenta de su frustración con el proceso de reforma política, de su rechazo a la Constitución, de su abandono de AP y, lo que es muy importante, para el autor como intelectual significado del franquismo, de su exclusión del diario $A B C$. A partir de entonces se tiene que refugiar en la creación de una revista de pensamiento, Razón Española, donde aglutinará a los cerebros ideológicos del franquismo: Millán Puelles, Fueyo Álvarez, Luis Suárez o José Luis Comellas.

Si los franquistas ideológicos rompen por la derecha, los sectores provenientes de la oposición moderada al franquismo se encuentran sin partido político. Desaparecida la UCD algunos optan por vincularse al CDS, otros apoyan la llamada «operación Roca», pero son muchos los que se encuentran sin un instrumento político que exprese su posición ideológica. Al igual que les había ocurrido a los democristianos que siguieron a Joaquín Ruiz-Giménez en su aventura electoral en 1977, se encuentran que no son socialistas pero tampoco conservadores. Son liberales pero el liberalismo no logra articular un partido propio en aquellos años ochenta. Los que más se acercaron a la posición liberal fueron los

2 Juan Francisco Fuentes, Adolfo Suárez. Biografia Politica, Barcelona, Planeta, 2011.

3 Gonzalo Fernández de la Mora, Rio Arriba, Barcelona, Planeta, 1995. 
miembros del CDS y, aún así, a principio de los años noventa tuvieron que desistir y el partido dejó de ser una opción electoral.

\section{La perspectiva liberal}

La desaparición de la UCD planteó una situación que afectó tanto a los liberales como a los socialistas. Los liberales se encuentran sin representación política y muchos de ellos consideran que el partido llamado a representar esa posición, a encarnar esas ideas, a defender esos ideales, es el PSOE. Creo que en todo este tema tiene una enorme importancia el papel jugado por el diario El País. En torno a este periódico se juegan muchos de los contenciosos intelectuales del período. Como sabemos en la evolución del diario se produce una transformación considerable en relación a los primeros objetivos.

Mari Cruz Seoane y Susana Sueiro han estudiado esta evolución mostrando que en un primer momento el referente intelectual del diario era el grupo de Revista de Occidente ${ }^{4}$. La evolución de los acontecimientos provocará un cambio muy significativo: no serán los orteguianos los que lideren el periódico. Es bien significativo que Julián Marías abandona el diario para acabar recalando en $A B C$. Así El País aparece como el heredero del mundo progresista liberal, como el heredero de Ortega, pero de un orteguismo que considera, como por cierto había propuesto el joven Ortega, que el partido llamado a europeizar España debía ser el partido socialista. Dicho de otra manera, que el PSOE tiene que compatibilizar su carácter de partido de clase con una tarea que va más allá del mundo obrero, con una tarea de vertebrar la nación y de alcanzar la europeización de España.

A partir de la desaparición de UCD el periódico se lanza a esta tarea, apoya decisivamente al partido socialista en su papel, pero a la vez muestra los límites que nunca deben ser traspasados. En todo el proyecto de modernización de la sociedad española, de consolidación de la democracia, de subordinación del poder militar al poder civil, de secularización de la sociedad y de inserción en Europa el discurso del diario El País no defiende nada distinto a lo que pensaban muchos de los representantes del pensamiento social cristiano, o del liberalismo progresista que se habían quedado sin partido. Donde estaba la dificultad era en lograr que ese programa de la burguesía democrática fuera aceptado, asimilado y apoyado por el PSOE.

Existían circunstancias que abonaban esa posibilidad. Tras el 23 de febrero de 1981 y tras la desaparición de la UCD, la necesidad de que alguien se hiciera cargo del país, la necesidad de volver a evitar tentaciones golpistas y de consolidar la democracia, había provocado que el triunfo electoral del PSOE constituyera un auténtico plebiscito democrático. Nada parecido se volvería a repetir en las elecciones posteriores (incluso cuando el PSOE en 1986 y el PP en el 2000 alcanzaron la mayoría absoluta). Aquel plebiscito a favor del PSOE ponía en primer plano el problema que tenía el partido socialista: ¿cómo hacerse cargo de las tareas de democratización, de modernización, de inserción en Europa y a la par, cumplir con su tarea como partido representante de los intereses de los trabajadores? Volvía a hacerse presente el viejo problema de aunar las dos tareas de los partidos socialistas: la realización de las reformas que demandaba el mundo liberal-laico y la representación de los intereses del mundo sindical. Así como en los gobiernos de Zapatero se ha producido una neutralización del conflicto social hasta que se da el giro en la política económica en

4 Mari Cruz SeoAne y Susana Sueiro, Una historia de El País y del grupo Prisa, Barcelona, Plaza y Janés, 2004 . 
mayo del 2010, los conflictos con los sindicatos son una constante desde el primer gobierno de Felipe González. Estamos en un momento donde todavía existía la llamada «familia socialista» $y$, por tanto, la vinculación entre partido y sindicato era muy estrecha ${ }^{5}$.

El papel del diario El País será decisivo para ir conformando una opinión pública favorable a las tesis del gobierno frente a las reclamaciones de los sindicatos. Para vestir intelectualmente este apoyo a las posiciones del liberalismo económico y en contra de las reivindicaciones de los sindicatos, se irá produciendo una legitimación intelectual en la que jugarán un papel esencial intelectuales que durante la dictadura habían pertenecido al Partido Comunista.

Para legitimar la función histórica que había propuesto el joven Ortega, era imprescindible presentar como algo arcaico, como algo obsoleto, como algo anacrónico, todo lo que significara la continuidad del proyecto socialista clásico. Había que apoyar el abandono de la definición marxista del partido socialista, situar a los sindicatos como organizaciones corporativas preocupadas únicamente por los intereses sectoriales y, lo que será muy importante, legitimar la permanencia de España en la OTAN. En todo este proceso juega un papel esencial El País. Hasta el punto de que creo es imposible comprender lo que ocurre en todo el mundo político entre 1982 y 1986 atendiendo únicamente a lo que se vive en los congresos del PSOE o en los debates parlamentarios, sin tener en cuenta la enorme importancia tiene la legitimación intelectual de las decisiones que se fueron tomando en el ámbito de la política.

A la pregunta de cuál fue el papel de los intelectuales, hay que responder, a mi juicio, de una forma clara: tuvieron un papel muy relevante a la hora de legitimar la transformación de la izquierda española. Por decirlo con el título de un libro donde El País recogía los editoriales más importantes fueron decisivos para que la izquierda gobernante entendiera que era prioritario Guardar la linea ${ }^{6}$.

\section{La perspectiva socialista}

Si esta perspectiva hegemónica del diario El País tiene consecuencias en el mundo liberal (la marcha de Julián Marías al diario $A B C$ sería la más significativa), lo mismo ocurre en dos mundos que no se sienten representados por el diario hegemónico: me refiero al mundo católico y al mundo socialista.

El mundo católico se encuentra con una situación peculiar. No prospera un partido democristiano, no tiene éxito una prensa católica y no se encuentran representados en el laicismo liberal que representa El País. Para constatar esta versión de lo vivido en aquellos años son muy recomendables la lectura de dos obras de signo bien distinto: me refiero a las memorias de Fernando Savater Mira por donde, y a la obra de Olegario González de Cardedal, Teología en España: 1959-20097. Justamente lo que le parece bien a Savater es lo que le parece mal a Olegario. Es decir la defensa del hedonismo, del agnosticismo, del liberalismo permisivo que encarna muy bien el diario contrasta con la crítica de Olegario a la falta de sensibilidad ante los temas religiosos, culturales, históricos, a la entraña del pensamiento cristiano.

5 Antonio Garcia Santesmases, Nicolás Redondo: historia, memoria y futuro, Madrid, Fundación Francisco Largo Caballero, 2007.

6 VVAA, Guardar la linea, Madrid, Ediciones El Pais, 1986.

7 Fernando Savater, Mira por dónde, Madrid, Taurus, 2003; Olegario Gónzalez de Cardedal, La teologia en España (1959-2009), Madrid, Ediciones Encuentro, 2010. 
Esa desazón de los teólogos taranconianos ante la evolución de la cultura de los ochenta es pareja al malestar que van a vivir las organizaciones sindicales. Diríamos que la tarea de vertebrar a la nación tenía como consecuencia negativa la desorientación de las bases católicas que no encontraban su lugar y la perdida de referencias de sectores de la izquierda que no sabían a que atenerse ante una evolución ideológica tan acelerada por parte del socialismo gobernante.

Es en este contexto en el que hay que ubicar las posiciones de tres intelectuales que van a marcar tres referencias importantes en el período: José Luis L. Aranguren, Enrique Tierno Galván y Manuel Sacristán. Los dos primeros van a marcar puntos de referencia en el mundo socialista y el tercero va a ser decisivo a la hora de configurar intelectualmente una izquierda alternativa. Vayamos con los dos primeros.

Enrique Tierno Galván juega un papel intelectual muy relevante entre los años 1975 y 1979. A partir de su llegada a la alcaldía de Madrid su papel disminuye. En los primeros años de la transición, Tierno representa al intelectual socialista que ha sufrido las penalidades del franquismo, que ha sido expulsado de la cátedra y que trata de encarnar un socialismo de izquierda, un socialismo de inspiración marxista. No había sido esa la posición de Tierno a lo largo de toda su vida intelectual. Tierno es un vencido de la guerra civil, que tiene que sobrevivir en un mundo de vencedores, donde era muy difícil representar una posición intelectual distinta al mundo de los católicos, o al mundo de los falangistas. Con muchos de aquellos católicos que evolucionan en los años sesenta Tierno establecerá relaciones de cooperación en la lucha contra la dictadura, al igual que con los falangistas que han evolucionado hacia el liberalismo.

Cabos Sueltos es, por ello, una obra decisiva para entender la cultura del antifranquismo. Es una obra que aparece en 1981 y que no tuvo la importancia que merece porque en aquel contexto las prioridades eran otras: había que consolidar la democracia y preparar la alternativa a un gobierno que se caía a pedazos como le ocurrió a la UCD. Había otra razón. Tierno había sido derrotado en su esfuerzo por hacerse con la hegemonía del socialismo español y había sido estigmatizado con los representantes de la primera Izquierda Socialista tras los congresos socialistas vividos en el año $1979^{8}$.

Los que habían apostado por mantener las señas de identidad de la Izquierda socialista de inspiración marxista habían quedado en minoría y Tierno fue recluyéndose en su trabajo en la alcaldía. Muchos de los militantes de su Partido Socialista Popular (PSP) no se incorporaron al PSOE o acabaron abandonándolo, otros optaron por incorporarse al CDS, como Raúl Morodo. Todo ello ha provocado que el legado intelectual de Tierno haya quedado desdibujado para muchos. Su temprana muerte en enero de 1986 provoca que se recuerde más su gestión como alcalde que al profesor conocedor de los clásicos del pensamiento político, al defensor del agnosticismo, al propulsor de un socialismo anticapitalista.

Sin embargo, hay que decir que en los años anteriores a la transición, y durante los años de la transición, los discípulos de Tierno, de Aranguren y de Sacristán conforman la generación más importante de intelectuales antifranquistas. Me refiero a discípulos de Tierno como Elías Díaz, Raúl Morodo, Pedro de Vega o Fernando Morán; a discípulos de Aranguren como Javier Muguerza, Pedro Cerezo, Ignacio Sotelo o Luis Gómez Llorente y a discípulos de Sacristán como Juan Ramón Capella, Francisco Fernández Buey, Jacobo Muñoz o Toni Domenech.

8 A. Garcia Santesmases, Repensar la izquierda, Barcelona, Anthropos, 1993. 
Las posiciones del Tierno de los últimos años del franquismo no se pueden entender sin comprender el impacto que tuvo el marxismo -las distintas lecturas del marxismo- en los intelectuales de distintas generaciones a partir de los sucesos del 68. Un impacto que afecta tanto a los que viven la caída de las últimas esperanzas en la evolución del socialismo real después de los sucesos de Praga, como a los que perciben los límites de la sociedad de consumo, de la sociedad del bienestar, de la alienación cultural del capitalismo avanzado, a partir de la irrupción del movimiento estudiantil.

Esa cultura vinculada al nacimiento de los nuevos movimientos sociales, a la apuesta por una «nueva izquierda», por un socialismo anticapitalista, está muy presente en los debates que suscitan las obras de Aranguren (pensemos en El marxismo como moral), de Tierno (pensemos en su Antología de Marx) o en los escritos breves -a menudo como prólogos a traducciones- de Manuel Sacristán. El hecho es que esa cultura va siendo abandonada por la izquierda mayoritaria y encuentra acogida en las palabras, en los mensajes, en las actitudes de estos intelectuales que siguen jugando un papel de referentes para los que no aceptan el proceso acelerado de desideologización.

Tierno que no puede seguir dedicando su tiempo a la vida intelectual desde la alcaldía no deja de hacer caer, de tanto en tanto, mensajes a favor de una cultura de la paz, a favor la razón de la ciudad frente a la razón del Estado y en la defensa de la utopía frente al posibilismo. Pero los requerimientos del trabajo de la alcaldía y el saber que él ya no tiene un partido propio, ni tiene el liderazgo dentro del socialismo español, hacen que procure espaciar muchos sus intervenciones. Su propia gestión como alcalde le da un reconocimiento público y una satisfacción que probablemente nunca hubiera imaginado.

Esta retirada de Tierno es la que refuerza aún más el papel de Aranguren en estos años, especialmente en los años del gobierno socialista. Hay que tener en cuenta que existe una gran diferencia entre el debate de los años $1975-78$ y las prioridades del periodo 1982-86. En el primer momento se trata de transitar a la democracia y de elaborar una Constitución. En el segundo momento este designio ya se ha cumplido y los protagonistas de aquel momento pasan a un segundo plano: Suárez reducido a un partido que tiene dos diputados; Santiago Carrillo, como ahora veremos, reducido a cuatro escaños y los ponentes constitucionales atareados en la presidencia del congreso (Gregorio Peces Barba), en liderar la oposición de la derecha conservadora (Manuel Fraga) o en propiciar un nuevo partido (Miquel Roca). Los debates constitucionales parecen haber quedado atrás aunque volverán con gran intensidad años después.

En este contexto el debate sobre la integración de España en Europa y sobre la permanencia en la OTAN adquieren una intensidad intelectual que no es sencillo de explicar a las generaciones que no vivieron aquellos tiempos. El debate sobre Europa apenas tuvo incidencia en la opinión pública, pero la adhesión fue ratificada por unanimidad en el parlamento. Parecía que también aquí el designio orteguiano se hacía realidad: España era el problema y Europa la solución. España había encontrado su sitio ${ }^{9}$.

Cuesta entender y explicar aquella polarización emocional porque hoy Europa sí es un problema, hoy asistimos a una crisis del modelo social europeo, pero, en los años ochenta, Europa era la esperanza de la modernización y la OTAN era el recuerdo de la dictadura de Franco. Europa era la esperanza de lo que no habíamos podido ser, no habíamos podido incorporarnos a las democracias que constituyen el consenso postotalitario y que dan vida a los años de la época de oro de la socialdemocracia. No lo logramos en 1945 al finalizar

9 Fernando Moran, España en su sitio, Madrid, Plaza y Janés, 1990. 
la Segunda Guerra mundial. La Europa que nos había abandonado en la Guerra civil, con la política de no intervención, nos volvía a abandonar apuntalando la dictadura de Franco. En ese apuntalamiento tenían una especial responsabilidad los distintos gobiernos norteamericanos.

Esta percepción estaba muy arraigada en la opinión pública española que no tenía el recuerdo de unos Estados Unidos liberadores del nazismo sino de una superpotencia que había apuntalado la dictadura de Franco. Por ello para conseguir la ratificación de la permanencia de España en la OTAN se necesitaba acudir a otro tipo de legitimación intelectual. No siendo posible acudir al recuerdo a un pasado de liberación, era imprescindible insistir en que Europa y Estados Unidos eran lo mismo y que si se quería estar en el mundo económico, cultural y político había que vincularse al mundo militar. Y añadir que ya que España tenía bases militares era preferible mantener una relación multilateral dentro de la OTAN que seguir anclados a la relación bilateral con los Estados Unidos. Ello significaba aceptar un designio de que todo estaba enlazado que había combatido el PSOE en la oposición.

El debate intelectual acerca de nuestro pasado y de la herencia del periodo dictatorial se conectó con el debate que agitaba a Europa en el mundo anterior a la Perestroika de Gorbachov, en el mundo de la carrera de armamentos, la tensión entre los bloques militares y el miedo a un choque entre las dos superpotencias. Hoy parece lejano aquel miedo a una guerra nuclear cuando hemos visto, en los años noventa y en el siglo veintiuno, la vuelta de guerras condicionadas por los sentimientos religiosos. No era ese el mundo de principio de los ochenta (aunque ya se hubiera producido la revolución de Jomeini en Irán y hubiera una guerra extraña entre Irán e Irak, y aunque también viviéramos la invasión soviética de Afganistán).

Lo que movía a los sectores pacifistas en Europa occidental era el intento de conectar la detención de la carrera de armamentos con el esfuerzo por apoyar la democratización de los países del Este. Un esfuerzo a favor del desarme y de la libertad que sería muy poco reconocido cuando se produjo la caída del comunismo en los países del Este. En todo este debate jugó un papel importante, como referente intelectual y moral, José Luis L. Aranguren. Atrás quedaban los debates del inicio de la transición entre los intelectuales orgánicos y los intelectuales que volaban libres de ataduras, para subrayar su independencia. El intelectual orgánico, en el sentido gramsciano, se vinculaba a un partido que operaba como intelectual colectivo y que luchaba contra la hegemonía cultural dominante. Pero una vez que el PSOE había abandonado el marxismo, ese modelo de intelectual quedaba atrás, mientras que la tesis de Aranguren ganaba adeptos. Aranguren había reivindicado siempre la soledad del intelectual, su apoyo solidario pero distante, su esfuerzo por no identificarse con la política de poder.

Esa disidencia moral conectaba muy bien con sectores intelectuales desencantados con el realismo de los años ochenta, con grupos insatisfechos con la evolución de la transición política y con movimientos cristianos que no se sentían reconocidos ni en la deriva neoconservadora del woytilismo ni en el laicismo liberal de la cultura hedonista dominante. Esos grupos vieron en Aranguren el referente intelectual con el que identificarse; vieron al hombre que se resiste a abandonar el clima utópico de los años sesenta y que se acerca con simpatía a los movimientos juveniles en torno a la desobediencia, la objeción de conciencia, y la insumisión al servicio militar.

Aranguren encarna muy bien esa disidencia moral, que se siente lejos del poder, que le pide al intelectual que mantenga una reserva moral frente al realismo, que no abdique de la esperanza de un mundo distinto. Esa encarnación de la disidencia no era muy bien recibida 
por muchos intelectuales que habían cambiado, que consideraban que toda aquella reserva moral, era una nostalgia del antifranquismo que no conducía a nada, que era el momento de asumir la política con mayúsculas como una actividad que exigía asumir las consecuencias del acceso al gobierno. La izquierda había soñado demasiado acerca del futuro mientras permitía que la derecha gestionara el presente. Era el momento de invertir las tornas y de mostrar que la izquierda estaba dispuesta a gobernar con todas las consecuencias y sin complejos $^{10}$.

Esa fue la posición dominante en la izquierda mayoritaria de aquellos años y a contribuir a ella jugó un papel esencial, como decíamos anteriormente, el diario El País. Al señalar los límites que no debían ser traspasados, el periódico establecía un filtro intelectual muy amplio: no eran convenientes políticamente ni el socialismo de izquierda dentro del PSOE, ni la contestación sindical, ni la resistencia moral de los intelectuales disidentes, ni las posiciones de una izquierda alternativa que comenzaba entonces a fraguarse.

\section{La emergencia de una izquierda alternativa}

Así como Aranguren representaba muy bien la postura del intelectual que vota al PSOE en 1982, pero que no le sigue en el referéndum de la OTAN de marzo de 1986, son otros los referentes intelectuales que van a llenar el hueco que se produce tras la debacle del comunismo español en 1982 al quedar reducido el PCE a cuatro diputados. De nuevo, aquí cuesta conectar con aquellos tiempos porque hoy discutimos mucho más de lo ocurrido entre 1975 y 1978 -es decir, analizamos las luces y las sombras del proceso constitucional y los problemas vinculados a la memoria y al olvido de la represión de la dictadura franquista-, que de lo que vivimos en el período siguiente.

Sobresale en este contexto la aportación de Manuel Sacristán, quien muere en agosto de 1985, meses antes del referéndum de la OTAN en marzo de 1986. Su influjo es notable en la evolución del postcomunismo español. A lo largo de la transición son muchos los intelectuales españoles que transitan del ámbito del comunismo al socialismo democrático o al liberalismo progresista. Hacíamos referencia anteriormente al caso de intelectuales -pensemos en Javier Pradera, Manuel Azcarate o Eduardo Haro Tecglen- que juegan un papel muy relevante en la andadura del diario El Pais, que visualizan muy bien este tránsito de una militancia comunista, en la época del antifranquismo, a una inserción en el periódico liberal progresista. También se puede recordar el caso de Fernando Claudín, que fue director de la Fundación Pablo Iglesias, o de Jorge Semprún o de Jordi Solé Tura, que ocuparon la cartera de ministros de cultura en distintos gobiernos socialistas.

No es el caso de Sacristán. No pertenece a la generación de jóvenes militantes comunistas vinculados a la experiencia de la Guerra civil, como es el caso de Azcárate y de Claudín, de Santiago Carrillo o de Adolfo Sánchez Vázquez. Sacristán nace en una familia de los vencedores de la guerra civil, es un joven falangista en los años cuarenta y se acerca al comunismo cuando ya cuenta treinta años, tras una estancia en Alemania. Josep María Castellet ha descrito muy bien a ese Sacristán juvenil en un libro reciente ${ }^{11}$. A través de esta obra, de la gran biografía de Juan Ramón Capella y del trabajo verdaderamente encomiable de Salvador López Arnal tenemos suficientes elementos para que una figura de este relieve

10 Ignacio Sotelo, Los socialistas en el poder, Madrid, Ediciones EI País, 1986.

11 Josep María Castellet, Ilustrados, seductores y visionarios, Barcelona, Anagrama, 2010. 
no se pierda en el olvido, para comprender porque fue, en palabras de Castellet, un seductor, visionario e ilustrado ${ }^{12}$.

En los años que estamos analizando, Sacristán aparece en dos momentos claramente diferenciados. En el primero, asociado a su vinculación a la revista Materiales, tenemos a un Sacristán que se propone establecer una crítica de izquierdas al eurocomunismo. Una tesis que mantendrá entonces y que resume su pensamiento es la tesis de que no procede engañarse acerca de la correlación de fuerzas, pero que no hay que desnaturalizarse abdicando del ideal emancipatorio ${ }^{13}$.

En un segundo momento, que podemos fechar a partir de 1979, Sacristán cambia de interlocutor. Ya no se trata de dirigirse a la base del partido comunista y vincularse a los debates internos acerca de la pervivencia del leninismo o de la plausibilidad del eurocomunismo, sino de plantear la necesidad de una alianza entre el movimiento obrero, el movimiento ecologista, el movimiento por la paz y el movimiento feminista. Esta segunda perspectiva es la que va a permitir que la revista que Sacristán funda en 1979 sea uno de los referentes más importantes en todo el debate sobre la permanencia de España en la OTAN. La revista Mientras tanto aparece así como el referente intelectual de una nueva forma de entender la política vinculada a los nuevos movimientos sociales. Al conectar con ese público movimentista, insumiso, pacifista, se produce una salida distinta a las que habíamos mencionado anteriormente en otros intelectuales. No son intelectuales comunistas que a partir de un determinado momento abandonan el PCE y se acercan al PSOE o a los órganos de la prensa liberal, sino que se sitúan en un mundo donde van a confluir con los movimientos Anti-OTAN y van a aparecer como portavoces de la preocupación por el ecologismo político. Esa posición del grupo de Sacristán tendrá una gran influencia tanto en los avatares de la izquierda social (pensemos en el papel jugado por Rafael Grasa en aquellos años), como posteriormente en la evolución de Izquierda Unida (IU) en la época de Julio Anguita (hasta el punto de que Víctor Ríos, discípulo de Sacristán, será el número dos de IU desde 1994 hasta el año 2000).

\section{Recapitulación}

La lectura que yo realizo de aquellos años es que los intelectuales juegan un papel muy importante a la hora de legitimar las decisiones políticas tomadas en el ámbito de la política institucional, o de combatir muchas de esas decisiones. Tanto los que quieren Guardar la línea como los que apuestan por la resistencia son muy relevantes para entender lo que está detrás del proceso político. Es una perspectiva que suele analizarse escasamente porque estamos acostumbrados a realizar una historia puramente política de sucesión de líderes, y de conflictos en el ámbito de la competencia entre partidos, sin tener en cuenta que sin la palabra aportada por los intelectuales no podemos entender ni el desconcierto de la derecha conservadora en los años 1982-1986 (basta recordar su comportamiento errático en el referéndum sobre la OTAN), ni el desasosiego del mundo católico, ni la ausencia de representación política en la que se encuentra el mundo del liberalismo progresista.

A partir del año 1986 se cierra el proceso de transición, se despejan las incógnitas, y establecido el marco de referencia, los debates durante años se vinculan al conflicto entre

12 Juan Ramón Capell. La práctica de Manuel Sacristán: una biografia política, Madrid, Trotta, 2005; id., Sin Itaca, Madrid, Trotta, 2011. Salvador Lopez Arnal y Pere De LA Fuente, Acerca de Manuel Sacristán, Barcelona, Destino Libro 1996.

13 Manuel SACristan, Intervenciones políticas, Barcelona, Icaria, 1985. 
los sindicatos y la política económica del gobierno. Será a partir de 1989 cuando empiece a resucitar políticamente el mundo conservador. Todo ello plantea dos problemas de los que quisiera ocuparme para finalizar.

En primer lugar, el debate que había centrado la reflexión política durante los años 1975 y 1978 se apagó en el periodo de 1982 a 1986. Todo el debate acerca de la forma de Estado (monarquía o república), en torno a la definición del propio Estado (laicidad, confesionalidad, aconfesionalidad) y acerca de la cuestión nacional (Estado de las autonomías, federalismo, centralismo), de alguna forma quedó aparcado durante aquellos años. Creo que a ello contribuyó enormemente la persistencia del fenómeno terrorista, y la aceptación de una relación especial entre el gobierno central y los gobiernos catalán y vasco. Eran los años en los que comenzaba la larga estancia de Jordi Pujol en el gobierno catalán, que durará 23 años, y la larga hegemonía del PNV en el gobierno vasco, desde 1980 hasta el 2009.

El tener definido el proceso de transición no implica que no hubiera discusiones acerca de la cuestión nacional, pero no tuvieron la virulencia intelectual que alcanzarían años después. Tampoco en el País Vasco, aunque fueran los años en los que existía un terrorismo más virulento. Las memorias de Fernando Savater son un buen testimonio de esa evolución. A partir de mitad de los años noventa los intelectuales jugarán un papel muy importante en el combate contra del nacionalismo etnicista, pero es un período muy posterior al que estudiamos en este texto ${ }^{14}$.

Lo mismo ocurre en el caso de Cataluña. Aquellos fueron años en los que el pujolismo dominaba el mundo político catalán y la política del gobierno catalán era la de no abrir ningún contencioso acerca del modelo constitucional ni acerca del marco aportado por el Estatuto de Cataluña. Fueron los acontecimientos de 1989-1991 en los países del Este los que fueron reabriendo la cuestión nacional, al visualizarse la posibilidad de constituir nuevos Estados-nación en Europa occidental, al moverse las fronteras, al percibir que lo que parecía imposible (la unidad alemana, la caída del bloque del Este) se hacía realidad.

Si en aquellos años se discutía poco sobre la cuestión nacional tampoco el debate estaba centrado en los temas de la recuperación de la memoria histórica republicana. Ahí sí que se podría decir que la historia había quedado, en aquel entonces, en manos de los historiadores. El tema de la memoria y del olvido volvería a aparecer ante la opinión pública a partir de los acontecimientos vividos en las dictaduras del Cono Sur latinoamericano, de las políticas de memoria de los Estados y de las demandas de justicia de las víctimas. Sólo a partir de esos sucesos comienza a aparecer la necesidad de iniciar un enjuiciamiento crítico sobre la transición: ¿hubo un exceso de olvido; se hizo justicia al legado del exilio; se mantuvo la necesidad de preservar la memoria republicana?

Esas preguntas, que hoy son habituales, no eran las que entonces nos haciamos. Entre las varias razones que se pueden alegar para interpretar aquel comportamiento hay una que puede ayudar a iluminar lo ocurrido en el mundo político y es analizando lo que pasó en el mundo intelectual. Si uno repasa el papel de los intelectuales importantes durante el periodo, observará que ninguno de ellos provenía del exilio. Y no porque no hubieran vuelto a España Francisco Ayala o Juan Marichal, o no nos visitaran otros como Adolfo Sánchez Vázquez. Ninguno de ellos tuvo el influjo que conservaron los referentes del antifranquismo, como Aranguren, Tierno o Sacristán. Fue injusto, pero creo que fue así.

14 Fernando SAvATER, op. cit., 2003. 
Si se me permite un recuerdo personal, es como si viviéramos inmersos en un marxismo intelectual, universitario, que no vivía la necesidad de conectar con la experiencia republicana de los años treinta. Estábamos más preocupados por las corrientes del pos68 y por los vaivenes del marxismo occidental que por los debates de los socialistas, los republicanos, los anarquistas o los comunistas durante los años de la Segunda República. Éramos más marxistas que republicanos. Hoy ocurre el fenómeno contrario. Hoy vuelve a estar de actualidad debatir sobre la vigencia de la Segunda República, sobre la necesidad de preservar el legado de aquellos años, sobre los límites del proceso de transición, sobre la necesidad de una memoria que haga justicia a las victimas.

Este fenómeno, que no se daba entonces, se produce en un contexto donde la derecha cultural, política y mediática tiene hoy una fuerza intelectual que no tenía en los años 1982-1986. Es, por ello, que recuperado el orgullo de su proyecto y dispuestos a defender su historia y su forma de entender la identidad nacional han producido un doble fenómeno que toda persona de izquierdas debería tener en cuenta y analizar con sumo cuidado. En primer lugar, se abomina de aquel pasado republicano presentándolo como un experimento totalitario, del que los españoles nos pudimos librar para nuestra ventura.

En segundo lugar, se abomina también de los intelectuales que fueron antifranquistas, recordando su pasado juvenil falangista o integrista. Al proceder de esta manera se intenta conseguir un doble resultado. La República no es ningún modelo moral del que haya que sentir nostalgia, fue un fenómeno execrable en el que nadie creía en la democracia, en la que todos fueron culpables, en la que nada hay que reivindicar porque de nada hay que enorgullecerse. Y la dictadura de Franco fue terrible pero también en ella todos fueron responsables, porque todos participaron de los beneficios del régimen o colaboraron en sus prácticas represivas. Al proceder de este modo no se establece una autocrítica al periodo republicano recordando las reflexiones de Azaña, de Prieto, de Juan Simeón Vidarte o de Julián Zugazagoitia, que habian establecido, en hora bien temprana, esa autocrítica. Se pretende demonizar a todo el régimen republicano.

Al realizar la segunda operación, al equiparar las conductas de unos y otros durante la dictadura, muchos de los grandes referentes antifranquistas -los que he citado aqui- quedan igualmente demonizados. Es sorprendente y trágico que esto se haga ante la pasividad y la ignorancia de muchos que no saben quién fue Aranguren, quién fue Sacristán, quién fue Tierno y aceptan silenciosamente, como si no fuera con ellos, los improperios de los que les quieren presentar como los Maestros franquistas de la izquierda ${ }^{15}$. Combatir tal desafuero debe ser una de las tareas prioritarias de una izquierda que no esté dispuesta a olvidar las raíces morales de la oposición a la dictadura y su contribución a la democracia española ${ }^{16}$.

15 Cesar Alonso De Los Rıos, Yo tenía un camarada, Barcelona, Altera, 2007

16 Antonio Garcia SANTESmases, "ZEran de barro nuestros maestros? Sobre las raíces morales de la oposición intelectual al franquismon, Ísegoria, $\mathrm{n}^{\circ} 31,2004$, p. 255-265. 